\title{
Déparasitage des volailles et vermifuges enzymatiques
}

\author{
par Z. DERBAL
}

\section{DÉPARASITAGE DES VOLAILLES}

Au Soudan français, les helminthiases mixtes (nématodes et cestodes) provoquent $50 \%$ de la mortalité des volailles.

Pour combattre ce fléau, nous avons cherché à mettre au point un système efficace, et pratique, associant le déparasitage périodique à certaines mesures prophylactiques : alimentation équilibrée désinfection des locaux et rotation des parcs à volailles.

Nos expériences ont commencé alu début de 1952 et ont été poursuivies jusqu'à la fin de l'année 1954; elles ont porté sur plus de 2.000 oiseaux.

Nous donnons ici un compte rendu sommaire de nos différents essais.

Chaque lot d'expérience, composé de 3 oiseaux âgés de six à huit mois provenant de parquets fortement infestés de cestodes et de nématodes, a été enfermé dans une cage garnic d'un fond grillagé permettant la récolte de tous les excréments dans un bac galvanisé.

Huit jours après l'administration de la dernière dose de vermifuge, toutes les volailles ont été sacrifiées.

Les produits suivants ont été utilisés :

Kamala + noix d'arec;

Didakène (capsules de tétrachloréthylèno chimi quement pur) ;

Didakene liquide;

Didakene huileux;

Bromhydrate d'arécoline en sol. 0.5\%;

Dicrolan en flacon (Institut de Sérothérapie de Toulouse);

Pelletiérine (alcaloïde de l'écorce de grenadier); Phénothiazine (thiodiphényl-amine);

Soufre:

Ail additionné à du salicylate de soude:

Essence de térébenthine.

Mode d'administration des médicaments :

I. - Kamala + noix d'arec : mélange à parties égales de kamala et de noix d'arec conditionné en capsules gélatinées contenant chacune $0,50 \mathrm{~g}$ de mélange.
Chaque animal a reçu $\mathrm{l} \mathrm{g}$ de ce mélange, soit 2 capsules par jour, pendant trois jours.

2. - Tétrachlorure d'éthylène pur (Didakène) : utilisé soit :

a) En liquide, après un jeûne strict de douze heures, on administre par le bec, très lentement et à l'aide d'une seringue, $1 \mathrm{~cm}^{3} \mathrm{du}$ produit. On répète l'opération au bout de quarante-huit heures.

b) En capsules de $1 \mathrm{~cm}^{3}$ : On donne quotidiennement et pendant deux ou trois jours de suite une capsule par oiseau.

C) Solution huileuse : On administre $1 \mathrm{~cm}^{3}$ de cette solution par le bec et $3-4 \mathrm{~cm}^{3}$ dans le cloaque à l'aide d'une seringue à embout prolongé.

3. - Bromhydrate d'arécoline : On administre par le bec $1 \mathrm{~cm}^{3}$ de solution à $0,50 \%$ de bromhydrate d'arécoline par jour pendant deux jours.

4. - Dicrolan (I.S.T.) (1) : On administre par le bec $1 \mathrm{~cm}^{3}$ de Dicrolan à base d'H.C.H. deux jours de suite.

5. - Pelletiérine : On administre par le bec en une seule fois $1 \mathrm{~cm}^{2}$ de pelletiérine suivi une heure après de $2 \mathrm{~cm}^{3}$ du mélange :

$$
\left.\begin{array}{l}
\text { - sirop de nerprun } \\
\text { - alcool à } 40 \%
\end{array}\right\} \widetilde{\mathbf{n}} \text {. }
$$

6. - Phénothiazine : On administre quotidiennement pendant trois jours une capsule gélatinée contenant $0,50 \mathrm{~g}$ de poudre de phénothiazine à $98,7 \%$ de produit pur.

7. - Phénothiazine + Kamala + Noix d'arec : On administre par le bec : le ler jour : 0,50 g de phénothiazine américaine, le $2^{\mathrm{e}}$ jour : deux capsules gélatinées contenant chacune $0,25 \mathrm{~g}$ de kamala et $0,25 \mathrm{~g}$ de noix d'arec.

8. - Didakène + Kamala + Noix d'arec : On administre le premier jour par le bec : $1 \mathrm{~cm}^{\mathrm{s}}$ de Didakène et 1 capsule de $0,50 \mathrm{~g}$ de mélange kamala i noix d'arec.

Les deux jours suivants : $1 \mathrm{~g}$ de mélange kamala + noix d'arec.

(1) Le Dicrolan de l'Institut de Sérothérapie de Toulouse contient l'isomère pur de l'H.C.H. 
9. - Soufre + Sulfate de soude : On donne I g de fleur de soufre par $\mathrm{kg}$ de poids vif puis, une heure après, $1 \mathrm{~g}$ de sulfate de soude en capsule.

Le deuxième jour : $1 \mathrm{~g}$ de fleur de souíre.

Le troisième jour, on répète le traitement du premier.

10. - Ail + Salicylate de soude : Pendant cinq jours, on mélange à la pâtée de l'ail et du salicylate de soude, à raison de $0,50 \mathrm{~g}$ de chaque par tête. Le traitement est répété une deuxième fois après dix jours de repos.

11. - Essence de térébenthine + Kamala + Noix d'arec : On administre deux jours de suite, le matin, à jeun, deux capsules de kamala + noix d'arec à $0,25 \mathrm{~g}$ par oiseau, et le troisième jour, au pistolet doseur, $4 \mathrm{~cm}^{3}$ du mélange suivant :

- essence de térébenthine... 1 partie,

- huile de paraffine ........ 3 parties.

Pour les jeunes oiseaux, on administre la moitié de la dose ci-dessus.

Le premier tableau résume les résultats.

Il ressort de ce tableau que, de tous les produits essayés, le mélange à parties égales de kamala, noix d'arec en capsules gélatinées de 0,50 g s'est montré le plus efficace et le moins dangereux, mais il a l'inconvénient de compromettre très sérieusement, sinon définitivement, la ponte el de ralentir la croissance. Il n'est pas sans danger pour les dindes et les pintades (1).

Dangereux pour les volailles, le tótrachlorure d'éthylène est, même à dose faible (1 $\mathrm{cm}^{3}$ par $\mathrm{kg}$ de poids vif), inutilisable pour les dindes et les pintades, ceci malgré l'opinion contraire formulée par certains auteurs (2).

M. le Professeur Lesbouyries (3) constate que le traitement du tæniasis est difficile. En effet, sous l'action des tænifuges, les anneaux de cestodes, peu adherents entre eux, se séparent et sont expulsés, mais la tête, enfoncée dans la paroi abdominale, se soustrait aisément à l'anthelminthique et reforme, par bourgeonnement, une nouvelle chaîne de proglottis.

Tue vermịuge idéal devrait avoir un très haut degré de toxicité pour les parasites intestinaux et être atoxique pour l'individu parasité (4).

Très rares sont les substances remplissant parfaitement ces conditions.

\section{Vermifuge enzymatique.}

Les efforts pour trouver un vermifuge répondant aux conditions précédemment citées aboutirent en 1951.

Le Docteur Schwab (*) de Munich, mit au point un

(*) Docieur Schwab - G.M.B.H. - Munchen 8. produit appelé Vermizym, produit que nous avons pu expérimenter au Centre fédéral de Recherches zootechniques de Bamako.

Les produits Vermizym du Docteur Schwab et Nematolyt de 'la firme Mack', tous deux à base de ferments enzymatiques (protéolytiques), sont utilisés depuis un certain temps en Allemagne, aussi bien en médecine humaine qu'en médecine vétérinaire.

Ammon (1950) a essayé in vitro le Nematolyt sur les vers intestinaux de l'homme.

Liebmann (1953) expérimente le Vermizym sur les parasites des animaux et trouve qu'ils sont vite dissous par ce produit. Il confirme également que l'action du ferment n'est pas nuisible à la flore bactérienne intestinale.

Depuis 1951, l'utilisation des ferments protéolytiques comme vermifuge des petits animaux a été généralisée en Allemagne. Les études histologiques sur l'influence de ces produits sur la muqueuse intestinale ont prouvé leur absolue inocuité, mème à grande concentration.

On donne couramment 1 g de Vermizym par $\mathrm{kg}$ de poids vif. Le produit peut être utilisé sur les animaux affaiblis, maigres, en gestation, sans aucun danger.

Capocaccia et Mastrandrea (6) ont même traité, avec succès, des malades en pleine crise d'ictère aigu, ceci sans provoquer de fatigue supplémentaire.

\section{Historique :}

Aux États-Unis (7), la méthode biologique de destruction des parasites intestinaux était connue dès 1920.

Wohmer (1911), Tschirsch (1920), et Chambers (1926) cités par Weise (1950) (8) ont découvert dans le jus de la papaye, fruit du Carica papaya ( « arbre à melons », papayer), un ferment enzymatique.

D'après Robbins (9) et Robbins et Lanson (10) (1934), on trouve dans la littérature américaine de nombreux travaux concernant les substances du lcchc de higueron. La sève des figuiers Ficus laurifolia, Ficus glabrate, Ficus carica, contient un ferment que Robbins a dénommé ficine.

L'enzyme contenu dans ce ferment a été identifié comme étant de la papaine, qui doit son nom au papayer. Cet arbre contient en effet plus de papaine que le figuier.

La papaïne peut dégrader les protides en acides aminés. Le Vermizym est un produit à base de papainne de Carica papaya. Il* perd son efficacité à $75^{\circ} \mathrm{C}$. La température optima pour son action se situe autour de $60^{\circ} \mathrm{C}$ et le $\mathrm{pH}$ optimum est autour de 5 .

Une large tolérance vis-à-vis du $\mathrm{pH}$ facilite son utilisation dans le milieu acide de l'estomac aussi bien que dans le milieu neutre de l'intestin. 


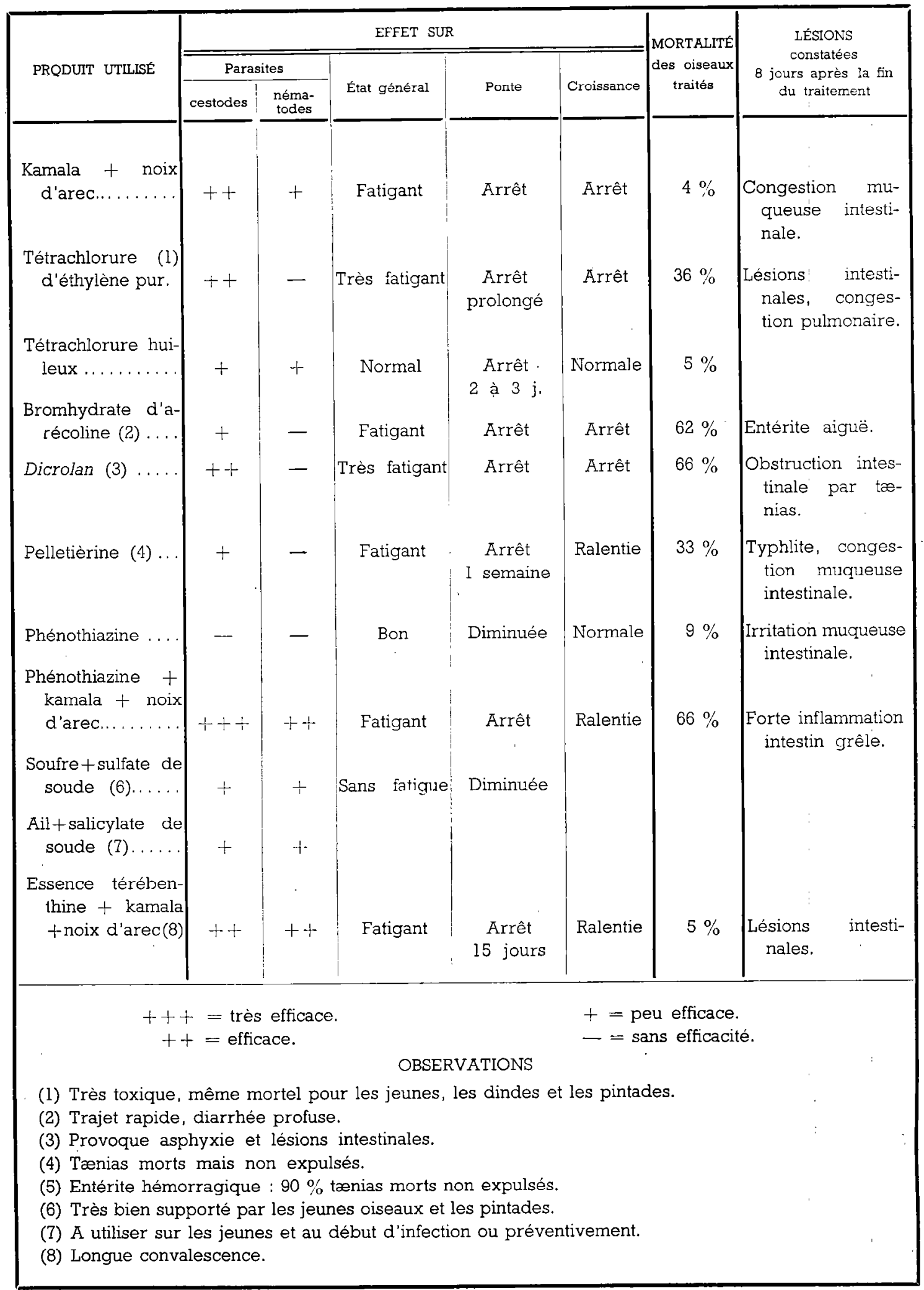


Les vers, protégés par leur cuticule, résistent à l'action de la trypsine et de l'érepsine, mais ils sont attaqués par le Vermizym qui entame et perce leur cuticule et ensuite les dissout. Cette deuxième phase est activée par la trypsine et l'érepsine.

Liebmann (7) a réalisé de nombreux essais in vitro notamment sur :

- Toxocara canis (chien).

- Strongylus vulgaris (cheval).

- Protostrongylus rufescens et nigrescens (mouton).

- Haemonchus contortus (mouton).

- Heterakis gallinæ (coq).

- Tænia hydatigena.

- Dipylidium caninum.

Ces différents parasites commencent à se dissoudre dans la solution à $4 \%$ de Vermizym au bout de une à quatre heures.

Effet sur les oufs : les cufs de Toxocara canis, libérés par macération no sont pas attaqués, la vie embryonnaire y continue.

Par contre, les œufs de Strongylus vulgaris, Dictyocaulus filaria, Protostrongylus rufescens et nigrescens, sont dissous.

Action sur les cestodes : Liebmann a prouvé que l'action était aussi efficace sur les vers plats que sur les vers ronds.

Ses essais portaient sur Tocnia hydatigena, Dipylidium caninum, Anoplocephala perfoliata.

Observations personnelles :

Nous basant sur nos expériences antérieures avec les différents produits décrits dans cette note, nous

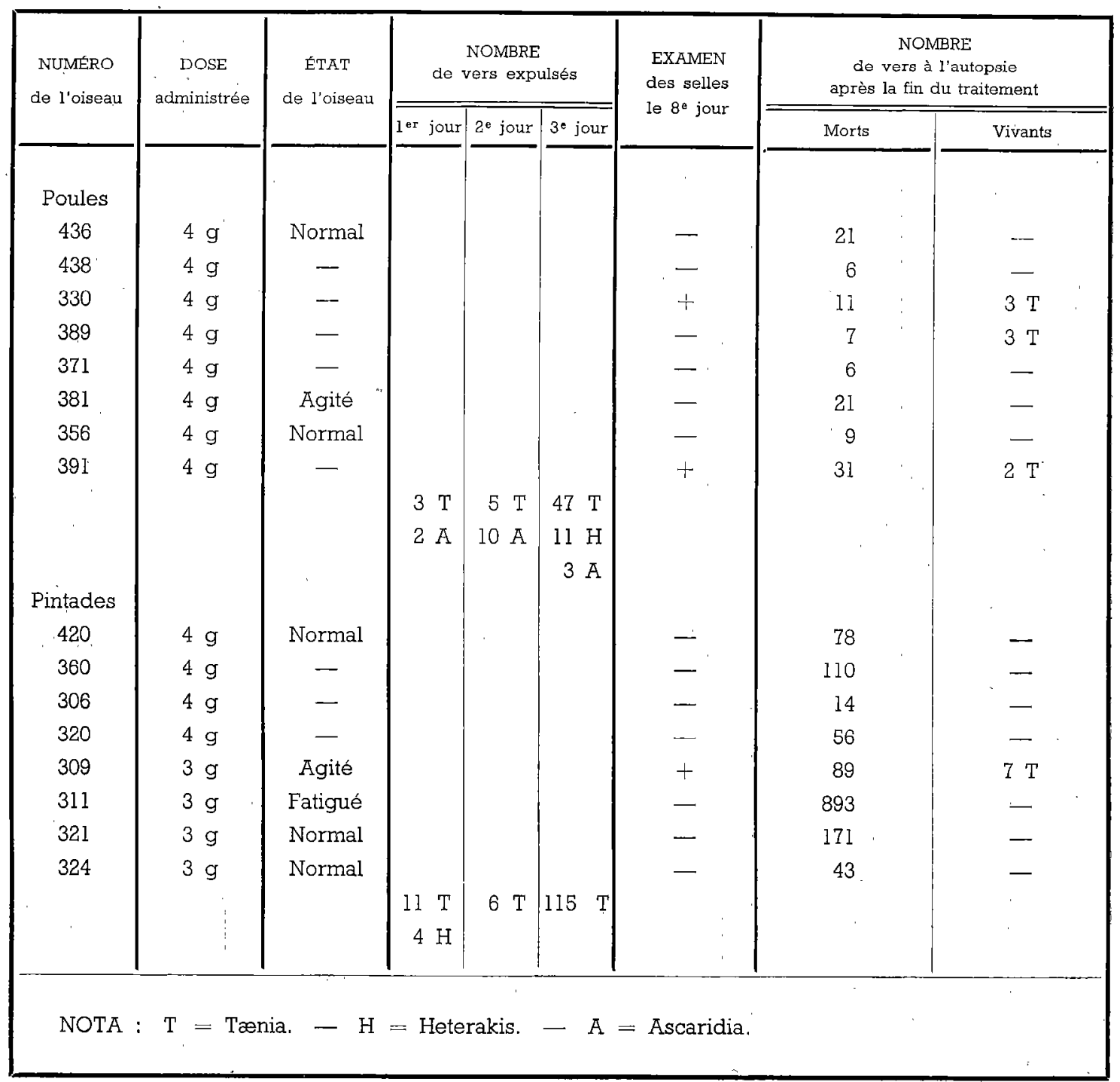


avons cherché à obtenir un déparasitage aussi complet que possible, sans altérer l'état général des oiseaux.

Le produit Vermizym a été essayé sur un parquet de poulettes fortement parasitées et sur des pintades qui s'étaient montrées particulièrement sensibles à tous les autres produits.

$\mathrm{L}$ 'expérimentation portait sur quatre lots, de quatre oiseaux chacun, dont deux lots de poulettes RhodesIsland et deux lots de pintades comprenant des adultes et des jeunes.

Administration du vermifuge. - Dans l'après-midi du jour précédant le traitement, on donne aux volailles un repas léger composé de légumes hachés (carottes de préférence).

Les dragées de Vermizym sont administrées le lendemain matin après un jeûne complet de douze à seize heures, à raison de $2 \mathrm{~g}$ par $\mathrm{kg}$ de poids vif le premier jour, et de $1 \mathrm{~g}$ par $\mathrm{kg}$ de poids vif les $2^{\mathrm{e}}$ et $3^{\mathrm{e}}$ jours.

Pendant la durée du traitement, il faut éviter toute alimentation riche en protides. On donne seulement une faible ration do mil.

Poulettes et pintades ont toutes très bien supporté le traitement sans manifester de fatigue; l'expulsion des vers a débuté les $2^{\mathbf{e}}$ et $3^{\mathbf{e}}$ jours.

Le second tableau résume les observations faites en cours de traitement.

Pratiquement, à l'examen des selles 'e $8^{\mathbf{e}}$ jour après le début du traitement, nous n'avons constaté que chez trois oiseaux la présence de quelques cufs de tænia.

L'autopsie de toutes les volailles, sacrifiées cing jours après la fin du traitement, montre une quantité importante de vers en état de macération avancée. Ces masses de vers sont entassées dans le grêle et plus particulièrement dans l'anse duodénale.

$D^{\prime}$ autres oiseaux (pintades) sacrifiées quinze jours après la fin du tıaitement n'avaient aucun parasite dans l'intestin. Les oiseaux vermifugés par la suite ont très bien supporté l'anthelminthique et ont continué à pondre et à croître normalement.

\section{CONCLUSION}

D'une efficacité parfois douteuse, la plupart des vermifuges utilisés habituellement pour le déparasitage périodique des volailles ont de multiples inconvénients :

- Ils affaiblissent les oiseaux;

- Arrêtent ponte et croissance :

- Irritent la muqueuse intestinale;

- Diminuent la vitalité de la flore bactérienne.
Les produits à base de ferments protéolytiques sont, au contraire, à la fois très efficace contre les vers intestinaux et inoffensifs tant pour la flore bactérienne que pour l'animal traité.

(Travail du Centre fédéral de Recherches zootechniques de Bamako. Soudan Français. A.O.F.).

\section{BIBLIOGRAPHIE}

1. Professeur I.-G. NEUMANN. - Parasites et maladies parasitaires des oiseaux domestiques, 1941, I vol. Vigot Frères, éditeurs, Paris, pp. 120121-137.

2. Edgar HUCH-BARGER and LESLIE ELLSWORTH GARD. - Diseases and Parasites of Poultry, 1949. 1 vol., Henry Kimpton, London, p. 317.

3. G. LESBOUYRIES. -- La pathologie des oiseaux, 1941, 1 vol., Vigot Frères, éditeurs, Paris, p. 665.

4. Docteur Martin MICK. - Neue Wege in der Behandlung der Eingeweidewiirmer. Deutsche Drogiste Zeitung, 1952, 12, Munchen.

5. Docteur J. BOCH. - Die Anwendung proteolytischer Fermente bei Nematodenbefahl der Pelztiere. Berl. und Munchener Tierärztliche Wochenschrift 1954, 17, 268, Verlag Paul Parey, Berlin SW, 68, Lindenstr. 44-47.

6. Docteur Livio CAPOCACCLA, Docteur Giovanni MASTRANDREA et Docteur Renato MORESCHI. - L'uso di un vermicida a base di fermenti proteolitici vegetali nell' infestazione da ascaridi. Archivo Italiano di Scienze Mediche Tropical et di Parasitologia, 1953, 34, 9 et 11 .

7. Professeur Docteur H, LIEBMANN: - Uber die Verwendung proteolytischen Fermente zur Bekämpfung der Nematoden des Haustiere. Berlin und Munchener Tierarztliche Wochenschrift, 1953, 2, 17.

8. Ch.-H. WEISE: - Uber die Wurmbekämpfung durch Enzyme. Medicinische Klinik, 1950, 35, 1096-1098.

9. ROBBINS - Cité par Weise. Journal Biol. Chem. $1934,106,7,5$.

10. ROBBINS a. LAMSON. - Cités par Weise. Journal Biol. Chem., 1930, 87, 251.

11. Em. PERROT. - Matières premières usuelles du règne végétal, 1948, 2 volumes, Masson, éditeur, tome 11, p. 1621. 